\title{
Antidepressant-like effect of mitragynine isolated from Mitragyna speciosa Korth in mice model of depression.
}

\begin{abstract}
Mitragyna speciosa Korth. leaves have been used for decades as a traditional medicine to treat diarrhea, diabetes and to improve blood circulation by natives of Malaysia, Thailand and other regions of Southeast Asia. Mitragynine is the major active alkaloid in the plant. To date, the role of mitragynine in psychological disorders such as depression is not scientifically evaluated. Hence, the present investigation evaluates the antidepressant effect of mitragynine in the mouse forced swim test (FST) and tail suspension test (TST), two models predictive of antidepressant activity and the effect of mitragynine towards neuroendocrine system of hypothalamic- pituitary-adrenal (HPA) axis by measuring the corticosterone concentration of mice exposed to FST and TST. An open-field test (OFT) was used to detect any association of immobility in the FST and TST with changes in motor activity of mice treated with mitragynine. In the present study, mitragynine at dose of $10 \mathrm{mg} / \mathrm{kg}$ and $30 \mathrm{mg} / \mathrm{kg}$ i.p. injected significantly reduced the immobility time of mice in both FST and TST without any significant effect on locomotor activity in OFT. Moreover, mitragynine significantly reduced the released of corticosterone in mice exposed to FST and TST at dose of $10 \mathrm{mg} / \mathrm{kg}$ and 30 $\mathrm{mg} / \mathrm{kg}$. Overall, the present study clearly demonstrated that mitragynine exerts an antidepressant effect in animal behavioral model of depression (FST and TST) and the effect appears to be mediated by an interaction with neuroendocrine HPA axis systems.
\end{abstract}

Keyword: Antidepressant-like activity; Corticosterone; Forced swimming test; HPA axis; Immobility time; Mitragyna speciosa; Mitragynine; Tail suspension test. 